\section{Is infection a factor in neonatal encephalopathy?}

\author{
Karin B Nelson, ${ }^{1,2}$ Anna A Penn ${ }^{3}$
}

In adults and in very preterm neonates, systemic infection is a well-recognised cause of encephalopathy. Although the manifestations of encephalopathy are very similar in the fetal inflammatory response and in acute hypoxic-ischaemic events, neonatal encephalopathy (NE) in term infants is more often attributed primarily to hypoxia-ischaemia rather than to infection. Is systemic infection an important etiological factor in NE in term and late preterm infants?

A striking 1999 case report $^{1}$ showed that placental infection with group B streptococcus can cause NE and that the clinical picture can closely mimic both immediate and later signs commonly assumed to indicate birth asphyxia. Many other reports describe associations of placental infections with encephalopathy in term neonates, suggesting that infection, or the resulting inflammation, can indeed underlie NE without an associated sentinel event causing asphyxia. This brief discussion considers the evidence of an infection-NE association, the difficulty of distinguishing infectious from noninfectious inflammation and the way forward.

Most of the evidence relating infection to NE has concerned infections identified during the admission for delivery, usually defined histologically in the placenta, or clinically. Histological evidence includes chorioamnionitis, which is inflammation on the maternal side of the placenta and funisitis on the fetal side, with infiltration of the umbilical cord with inflammatory cells. Clinical evidence of infection includes maternal fever in labour, uterine tenderness, purulent discharge or fetal tachycardia. A third approach to identifying placental infection is by microbiological evaluation. Unfortunately, results of these approaches are often not in agreement, ${ }^{2}{ }^{3}$ and multiple identification methods are rarely used in the same study.

${ }^{1}$ Children's Hospital National Medical Center, Washington, DC, USA; ${ }^{2}$ National Institutes of Health, NINDS, Bethesda, Maryland, USA; ${ }^{3}$ Fetal \& Transitional Medicine, Neonatology, Children's National Medical Center, Center for Neuroscience Research, Washington, DC, USA

Correspondence to Dr Karin B Nelson, 5524 Charles Street, Bethesda, MD 20814, USA; nelsonk@ninds.nih. gov

\section{HISTOLOGICAL CHORIOAMNIONOITIS}

McDonald et $a l^{4}$ compared placentas of 93 infants with $\mathrm{NE}$ with placentas of normal term controls $(n=816)$ and of random controls $(n=387)$, finding funisitis in $31.2 \%$ of NE placentas versus $5.4 \%$ and $4.4 \%$ of controls $(p=0.002)$. Chorioamnionitis and villitis were also significantly more frequent in $\mathrm{NE}$, especially in more severe grades of histological inflammation. Vasculitis, endothelial damage, fibrin deposition and thrombus formation may occur as a consequence of severe infection. Reports subsequent to McDonald et $a l^{4}$ have also associated severe placental inflammation involving fetal response with NE, especially if associated with thrombovascular lesions. ${ }^{5} 6$ Milder inflammation without fetal response was not associated with NE.

In a population-based Canadian registry, $^{7}$ chorioamnionitis was more frequent in the $34 \%$ of term-born children with cerebral palsy (CP) who had had NE as newborns, as compared with those with CP who had not manifested NE.

Even infants admitted for therapeutic cooling, selected because their neurological dysfunction was thought to stem from acute asphyxial injury, had high rates of inflammatory placental lesions. ${ }^{8-10}$ Histological chorioamnionitis is also associated with other risk factors for $\mathrm{NE}$ and $\mathrm{CP}$, including low Apgar scores. ${ }^{11}{ }^{12} \mathrm{~A}$ recent study not finding histologic chorioamnionitis to predict NE in term infants did not describe severity of inflammation or presence of a fetal response. ${ }^{13}$

\section{CLINICAL CHORIOAMNIONITIS}

Few large population-based studies of infection/inflammation and NE have had systematic placenta histology and therefore have employed clinical indicators as a surrogate. The most common indicator used is maternal fever in labour, associated in controlled studies with heightened risk of NE. ${ }^{14}{ }^{15}$ Clinical chorioamnionitis defined as maternal fever was twice as frequent as identified asphyxial birth events in neonates admitted for therapeutic hypothermia ${ }^{16}$ and is a frequent antecedent of low Apgar scores. ${ }^{17}{ }^{18}$ Neonatal seizures are a common feature of NE; a clinical diagnosis of chorioamnionitis, other maternal infection and isolated fever were all associated with increased risk of neonatal seizures. ${ }^{19}$ Perinatal stroke has also been associated with intrapartum fever. ${ }^{20}$

Maternal fever in labour has many potential causes, including intrauterine or systemic infection, dehydration, muscular effort or epidural anaesthesia. Epidural anaesthesia is associated with lower Apgar scores and heightened risk of neonatal seizures. $^{21}$ Whether epidural-related fever is regularly accompanied by chorioamnionitis is not well agreed. ${ }^{22} 23$

In a large population-based study, clinical markers of inflammation were associated with a 5.6-fold increase in risk of clinically diagnosed hypoxic-ischaemic encephalopathy (HIE) in neonates with later-recognised $\mathrm{CP}^{24}$ Clinical markers, although neither sensitive nor specific for infection/inflammation, permitted the tentative estimate that these contribute about $10 \%$ of clinically diagnosed HIE in infants with $\mathrm{CP}$ and $3 \%$ of total $\mathrm{CP}^{24}$ Term neonates with inflammatory markers who were neurologically asymptomatic in the first days of life were not at increased risk of CP. NE appears to be on a pathway from infection/inflammation to $\mathrm{CP}$.

\section{MICROBIOLOGICAL EXAMINATION}

It is usually assumed that chorioamnionitis and funisitis are the results of infection by microorganisms that directly cause neonatal sepsis or produce injurious systemic inflammation. For example, in early onset neonatal group B streptococci infection, neurological symptoms are common even without evidence of meningitis. ${ }^{25}$ In very preterm placentas, association with microbiological infection seems to be largely true, but for term placentas the evidence is weaker. Some reports have identified infective microorganisms in the placenta in NE, but culture-proven or histologically visible infection was rare in acute histological chorioamnionitis in placentas of term infants born to low-risk mothers. ${ }^{26}$ Significantly, there were indications of an inflammatory state in these women before the birth. $^{26}$ It remains uncertain how much of the inflammation associated with $\mathrm{NE}$ is infectious in nature.

\section{NON-INFECTIOUS INFLAMMATION}

Non-infectious inflammation in the placenta can apparently contribute to NE. The classic form of placental noninfectious inflammation is chronic villitis, defined by chronic inflammatory cells of maternal origin in fetal villi. In most cases, no infective organism is recognised and the condition is known as villitis of unknown aetiology (VUE). VUE is observed in about $10 \%$ of term placentas, 
apparently related to breakdown of maternal tolerance for the fetoplacental unit as parturition approaches.

Several controlled studies link highgrade VUE with increased risk of $\mathrm{NE}^{4} 610$ and with lesions on MRI imaging that are commonly attributed to asphyxial birth events. ${ }^{10}$ VUE is also associated with risk factors for NE and for CP, including prior fetal loss, fetal growth restriction, nonreassuring fetal heart rate patterns in labour, emergency surgical delivery, and severe acidosis in the absence of a sentinel event. ${ }^{27}$ VUE can thus mimic birth asphyxia and cannot be recognised without placental examination.

The link between NE and other noninfectious forms of inflammation, such as that related to maternal autoimmune disease, is largely unexamined.

\section{IMPLICATIONS}

Placental lesions are common in NE, suggesting that antenatal infection, inflammation and/or vasculopathy occur in some or much of NE, including that clinically described as hypoxic-ischaemic. Reliance on analyses that do not evaluate evidence of infection/inflammation or severity of inflammation may lead to an exaggeration of the role of intrapartum asphyxia as a singular cause for NE.

Therapeutic cooling has led to decreased mortality and morbidity in neonates with $\mathrm{NE}$, but unfortunately, only a minority of cooled babies benefit. ${ }^{28}$ Neonatal hypothermia was designed as an intervention for acute asphyxial injury following a sentinel event. Might placental inflammation and/or vasculopathy in NE limit therapeutic response to cooling? ${ }^{29}$ There is little evidence that cooling improves outcome in neonates with placental inflammation and/or vasculopathy; observations by Wintermark et $a l^{8}$ and others suggest that it may not.

No biomarkers have yet been identified that are highly specific to infectious versus non-infectious inflammation. Of the biomarker panels that exist, few predict outcome sufficiently well for clinical use. 'To date, no microbiological test, clinical sign or scoring system, or laboratory marker is able to safely distinguish between infected and uninfected infants. ${ }^{30}$ Ubiquitous use of antibiotics is likely to continue in NE for the foreseeable future.

Might treatment targeting immune processes and inflammation benefit some neonates with NE? How will we identify them? In the near future, much of our information about infants with NE will come from randomised trials that test cooling plus an additional therapeutic intervention (cooling+). Many of the adjunct medications being tested, such as erythropoietin and melatonin, have global anti-inflammatory properties in addition to their specific activities. ${ }^{31}$ Only the collection of data on infection/inflammation in enrolled infants and prospectively planning secondary analyses in upcoming trials will provide information on whether these medications offer additional benefit and if their anti-inflammatory properties contribute usefully to management.

In addition, novel therapeutics being developed in preclinical models, such as neurosteroids, growth factors and unique anti-inflammatory agents, should be assessed in current experimental models that use hypoxia and/or ischaemia to mimic term or near-term NE, and in peripartum inflammation models and in combination models ${ }^{32}$ that may better reflect the complexity of human NE.

Moving forward, cooling + trials should incorporate information on placental histology, bacteriology and molecular markers of placental infection, along with maternal and family history. Secondary analyses in those trials could test the hypothesis that severe inflammation of the placenta with fetal response is associated with $\mathrm{NE}$, and could attempt to distinguish infectious from non-infectious inflammation.

Recognising which depressed neonates have placental lesions, particularly funisitis and vasculitis which are highly associated with NE, may enable future cooling + trials to focus on infants most likely to benefit, while seeking additional or alternative treatments for neonates with evidence of vasculopathy, infection or non-infectious inflammation.

Contributors $\mathrm{KBN}$, a paediatric neurologist, wrote the main body of this article. AAP, a neonatologist and neuroscientist with special interest in the placenta, contributed a section and made useful suggestions concerning the remainder. AAP is partly supported by NIH Director's New Innovator Award (DP20D006457).

\section{Competing interests None.}

Provenance and peer review Commissioned; externally peer reviewed.

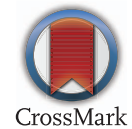

To cite Nelson KB, Penn AA. Arch Dis Child Fetal Neonatal Ed 2015;100:F8-F10.

Received 10 June 2014

Revised 28 July 2014

Accepted 30 July 2014

Published Online First 28 August 2014

Arch Dis Child Fetal Neonatal Ed 2015;100:F8-F10. doi:10.1136/archdischild-2014-306192

\section{REFERENCES}

1 Keogh JM, Badawi N, Kurinczuk JJ, et al. Group B streptococcus infection, not birth asphyxia. Aust N Z J Obstet Gynaecol 1999;39:108-10.

2 Queiros da Mota V, Prodhom G, Yan P, et al. Correlation between placental bacterial culture results and histological chorioamnionitis: a prospective study on 376 placentas. J Clin Pathol 2013;66: 243-8.

3 Smulian JC, Shen-Schwarz S, Vintzileos AM, et al. Clinical chorioamnionitis and histologic placental inflammation. Obstet Gynecol 1999:94:1000-5.

4 McDonald DG, Kelehan P, McMenamin JB, et al. Placental fetal thrombotic vasculopathy is associated with neonatal encephalopathy. Hum Pathol 2004:35:875-80.

5 Redline RW, O'Riordan MA. Placental lesions associated with cerebral palsy and neurologic impairment following term birth. Arch Pathol Lab Med 2000;124:1785-91.

6 Hayes BC, Cooley S, Donnelly J, et al. The placenta in infants $>36$ weeks gestation with neonatal encephalopathy: a case control study. Arch Dis Child Fetal Neonatal Ed 2013;98:F233-9.

7 Shevell A, Wintermark $P$, Benini $R$, et al. Chorioamnionitis and cerebral palsy: lessons from a patient registry. Eur I Paediatr Neurol 2014:18:301-7.

8 Wintermark P, Boyd T, Gregas MC, et al. Placental pathology in asphyxiated newborns meeting the criteria for therapeutic hypothermia. Am J Obstet Gynecol 2010:203:579.e1-9.

9 Chang T, Reyes C, Teng J, Placette J, et al. Neonatal encephalopathy, sentinel events, and the placenta. I Neonatal-Perinatal Med 2012;5:1-8.

10 Harteman JC, Nikkels PG, Benders MJ, et al. Placental pathology in full-term infants with hypoxic-ischemic neonatal encephalopathy and association with magnetic resonance imaging pattern of brain injury. J Pediatr 2013;163:968-95.e2.

11 Nelson KB, Ellenberg JH. Obstetric complications as risk factors for cerebral palsy or seizure disorders. JAMA 1984:251:1843-8.

12 Wong L, MacLennan AH. Gathering the evidence: cord gases and placental histology for births with low Apgar scores. Aust N Z I Obstet Gynaecol 2011:51:17-21.

13 Jenster M, Bonifacio SL, Ruel T, et al. Maternal or neonatal infection: association with neonatal encephalopathy outcomes. Pediatr Res 2014;76:93-9

14 Badawi N, Kurinczuk JJ, Keogh JM, et al. Antepartum risk factors for newborn encephalopathy: the Western Australian case-control study. BM 1998;317:1549-53.

15 Blume HK, Li Cl, Loch CM, et al. Intrapartum fever and chorioamnionitis as risks for encephalopathy in term newborns: a case-control study. Dev Med Child Neurol 2008:50:19-24.

16 Nelson DB, Lucke AM, McIntire DD, et al. Obstetric antecedents to body-cooling treatment of the newborn infant. Am J Obstet Gynecol 2014;211:155 e1-6.

17 Alexander JM, McIntire DM, Leveno KJ. Chorioamnionitis and the prognosis for term infants. Obstet Gynecol 1999;94:274-8.

18 Grether JK, Nelson KB. Maternal infection and cerebral palsy in infants of normal birth weight. JAMA 1997;278:207-11.

19 Glass HC, Pham TN, Danielsen B, et al. Antenatal and intrapartum risk factors for seizures in term newborns: a population-based study, California 1998-2002. J Pediatr 2009;154:24-8.e1.

20 Mann JR, McDermott S, Pan C, et al. Maternal hypertension and intrapartum fever are associated with increased risk of ischemic stroke during infancy. Dev Med Child Neurol 2013;55:58-64. 


\section{Leading article}

21 Greenwell EA, Wyshak G, Ringer SA, et al. Intrapartum temperature elevation, epidural use, and adverse outcome in term infants. Pediatrics 2012;129:e447-54.

22 Riley LE, Celi AC, Onderdonk AB, et al. Association of epidural-related fever and noninfectious inflammation in term labor. Obstet Gynecol 2011:117:588-95.

23 Dashe JS, Rogers BB, McIntire DD, et al. Epidural analgesia and intrapartum fever: placental findings. Obstet Gynecol 1999;93:341-4.

24 Mclntyre S, Blair E, Badawi N, et al. Antecedents of cerebral palsy and perinatal death in term and late preterm singletons. Obstet Gynecol 2013;122: 869-77.
25 Andersen J, Christensen R, Hertel J. Clinical features and epidemiology of septicaemia and meningitis in neonates due to Streptococcus agalactiae in Copenhagen County, Denmark: a 10 year survey from 1992 to 2001. Acta paediatrica 2004;93: 1334-9.

26 Roberts DJ, Celi AC, Riley LE, et al. Acute histologic chorioamnionitis at term: nearly always noninfectious. PloS one 2012;7:e31819.

27 Greer LG, Ziadie MS, Casey BM, et al. An immunologic basis for placental insufficiency in fetal growth restriction. Am J Perinatol 2012;29:533-8.

28 Jacobs SE, Berg M, Hunt R, et al. Cooling for newborns with hypoxic ischaemic encephalopathy. Cochrane Database Syst Rev 2013:1:CD003311.
29 Nelson KB, Mclntyre S, Badawi N, et al. Does aetiology of neonatal encephalopathy and hypoxic-ischemic encephalopathy influence the outcome of treatment? Dev Med Child Neurol 2014, In press.

30 Hofer N, Zacharias E, Muller W, et al. Performance of the definitions of the systemic inflammatory response syndrome and sepsis in neonates. J Perinat Med 2012;40:587-90.

31 Ofek-Shlomai N, Berger I. Inflammatory injury to the neonatal brain-What can we do? Front Biosci 2014:2:30.

32 Bolouri H, Savman K, Wang W, et al. Innate defense regulator peptide 1018 protects against perinatal brain injury. Ann Neurol 2014;75:395-410. 